\title{
Kirstin Ott, Berlins verschwundene Denkmäler. Eine Verlustanalyse von 1918 bis heute.
}

\section{Emmanuel Droit}

\section{(2) OpenEdition}

\section{Journals}

Electronic version

URL: https://journals.openedition.org/ifha/11557

DOI: 10.4000/ifha. 11557

ISSN: 2198-8943

\section{Publisher}

IFRA - Institut franco-allemand (sciences historiques et sociales)

\section{Electronic reference}

Emmanuel Droit, "Kirstin Ott, Berlins verschwundene Denkmäler. Eine Verlustanalyse von 1918 bis heute.", Revue de I'IFHA [Online], Date of review, Online since 07 December 2021, connection on 09 December 2021. URL: http://journals.openedition.org/ifha/11557 ; DOI: https://doi.org/10.4000/ifha. 11557

This text was automatically generated on 9 December 2021

(CIFHA 


\title{
Kirstin Ott, Berlins verschwundene Denkmäler. Eine Verlustanalyse von
} 1918 bis heute.

\author{
Emmanuel Droit
}

\section{REFERENCES}

Kirstin Ott, Berlins verschwundene Denkmäler. Eine Verlustanalyse von 1918 bis heute, Berlin, Lukas Verlag, 2020, 448 p., $36 €$. 
$\mathrm{Au}$ sein $\mathrm{du}$ champ de recherche interdisciplinaire désormais bien établi sur les études mémorielles, l'ouvrage de Kirsten Otto tiré d'une thèse de doctorat soutenu en 2016 à l'Université Humboldt de Berlin invite le lecteur à faire un pas de côté et à aborder l'enjeu de la mémoire monumentale à travers la notion de perte. Les monuments et les mémoriaux sont des vecteurs relevant de la «mémoire culturelle» (J. Assmann) mais en tant qu'objets matériels inscrits de manière visible et centrale dans l'espace public, ils peuvent être kidnappés, déplacés, transférés ou détruits. D'ailleurs, comme un clin d'œil à la célèbre formule de $\mathrm{T}$. Nipperday "Am Anfang war Napoléon », le livre s'ouvre sur le « vol» du quadrige que l'Empereur déboulonna et ramena à

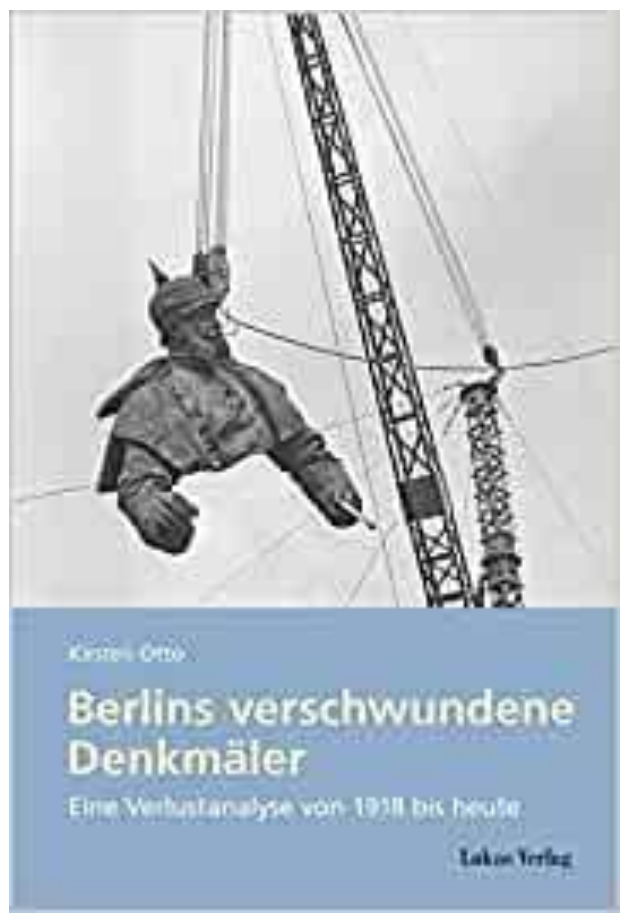
Paris en 1806.

L'historienne inscrit son analyse dans la tradition incarnée par la réflexion de Reinhart Koselleck sur l'injonction commémorative aux morts sous la forme de mémoriaux («Les monuments aux morts comme fondateurs de l'identité des survivants ", Revue de Métaphysique et de Morale, $\mathrm{n}^{\circ}$ 1, 1998, p. 33-61). Elle a choisi de centrer son analyse de la perte sur l'espace urbain de Berlin entre la chute de l'Empire wilhelminien en 1918 et la sortie de guerre froide. Le choix de la capitale allemande permet à K. Otto d'aborder les effets du "siècle des extrêmes" sur les monuments et mémoriaux des différents régimes politiques présents dans la ville-synecdoque de l'histoire du $\mathrm{XX}^{\mathrm{e}}$ siècle : la République de Weimar, les deux dictatures allemandes et la République de Berlin. L'objectif de ce livre richement illustré n'est pas de se livrer à une analyse singulière de chaque monument "perdu » mais bien de considérer globalement l'espace berlinois comme un microcosme dynamique à partir duquel les causes et les conséquences de la perte seront étudiées. Plutôt que de se concentrer sur les processus de création des monuments, K. Otto plonge le lecteur dans une histoire de la disparition topographique et mémorielle qui peut être provisoire ou définitive.

Le livre est structuré en deux grandes parties qui renvoient aux deux grandes catégories de l'analyse historique : l'événement d'un côté, la structure de l'autre. La première est consacrée de manière chronologique au destin de ces monuments qui disparaissent du paysage urbain de Berlin entre 1918 et aujourd'hui. Chaque régime politique fait l'objet d'une sous-partie, permettant de mesurer de manière diachronique la manière dont les césures politiques $(1918,1933,1945,1949,1990)$ ont un impact sur des statues, des mémoriaux. Les stratégies de damnation memoriae peuvent prendre la forme de mise à l'écart de bustes ou de statues de personnalités juives sous le $\mathrm{III}^{\mathrm{e}}$ Reich, de généraux prussiens sous la RDA.

La seconde partie du livre est plus structurel dans la mesure où elle retrace la " carrière " des monuments de leur conception à leur disparition en passant par des formes de réutilisation, de dégradation ou de réactivation. Elle a vocation à fournir un 
cadre théorique général qui pourrait s'appliquer à d'autres espaces urbains allemands ou internationaux. Chaque sous-partie lui permet d'aborder une dimension des monuments: les actions de vandalisme d'acteurs de la société civile exprimant une forme de contre-mémoire, les actions de transformation, de déplacement ou de démontage entreprises par les autorités politiques. L'auteur s'intéresse également à la mémoire de l'objet, du lieu et aux entreprises de réactualisation de cette mémoire culturelle. K. Otto souligne sans grande surprise le fossé entre les intentions initiales qui président à la construction d'un monument - à savoir l'inscription dans la durée et l'indestructibilité - et le devenir de ce dernier soumis aux aléas de la conjoncture politique. Ce résultat permet à l'auteur de contredire la thèse défendue au sein de la recherche historique par Martin Warnke selon laquelle l'iconoclasme est une pratique qui appartient à un passé révolu. Ce que rappelle $\mathrm{K}$. Otto, c'est que les révolutions, en tant que césures politiques, mais aussi les guerres ou les situations de guerre produisent beaucoup de disparitions de monuments : on détruit des statues au nom de la geste révolutionnaire de la tabula rasa, on utilise le matériau de monuments en période de guerre pour les besoins économiques. En tant que lieu concentrant tous les extrêmes du XXe siècle, Berlin constitue certainement un cas exceptionnel de par la régularité des changements politiques. Mais les débats actuels suite à la dégradation de la statue de Colbert devant l'Assemblée nationale le 23 juin 2020 par des membres du groupe " Anti-Néggrophobie » nous rappelle que la perception des monuments dépend à la fois du contexte politique et des transformations des cultures mémorielles dans des sociétés européennes où les enjeux identitaires contribuent à une fragmentation de la mémoire nationale.

INDEX

Subjects: Histoire culturelle

Chronological index: Epoque Contemporaine

\section{AUTHOR}

EMMANUEL DROIT

Sciences Po Strasbourg 